Brit. Heart f., 1968, 30, 432.

\title{
Cardiac Leiomyosarcoma: Primary or Secondary?
}

\author{
A. G. NICOL AND G. M. MCANDREW \\ From the Department of Pathology, University of Aberdeen, and Aberdeen Royal Infirmary
}

\begin{abstract}
Neoplastic involvement of the heart is not uncommon, but this case presents several features of unusual interest. Almost 10 years after removal of a malignant fibroid of uterus, the patient died as a result of cardiac involvement by a leiomyosarcoma. During the terminal illness difficulty was encountered in reaching a clinical diagnosis and, even after necropsy, a firm opinion as to the relation between the two malignant tumours of smooth muscle has proved impossible.
\end{abstract}

\section{Case Report}

Early Clinical Features. A 35-year-old multiparous woman was first seen in February 1954, complaining of frequent and increasingly heavy menstrual bleeding. No pelvic abnormality was found at that time and a normal endometrium was obtained on curettage. The patient's symptoms persisted and on December 6, 1955 laparotomy was performed and showed the uterus to be enlarged by several small fibroids. The left ovary was normal apart from a small follicular cyst which was punctured. The right ovary was enlarged and contained many follicular cysts. Total hysterectomy and right salpingo-oophorectomy were performed; a cervical fibroid $(6 \mathrm{~cm}$. diam.) which was growing out into the parametrial tissue caused some difficulty at operation but appeared to have been completely removed. Postoperative recovery was uneventful.

Histology of Uterine Tumour (Fig. 1a). The tumour consisted of interlacing bundles of smooth muscle fibres, but showed numerous groups of abnormally large cells. Some of these possessed atypical single nuclei with irregular chromatin masses while other cells were multinucleate. Van Gieson's method, Mallory's phosphotungstic acid haematoxylin, and other stains showed little collagen deposition, and cross-striation of muscle fibres was not detected on staining with Heidenhain's ironhaematoxylin. Only occasional mitotic figures were seen, but aberrant areas were present throughout the tumour which was reported as a leiomyosarcoma.
Subsequent Progress. After the operation the patient remained well and no pelvic abnormality was found at the time of her last regular review in August 1957. About the beginning of 1965 she began to complain of increasing lassitude, tiredness, and loss of weight, and early in May suffered a minor head injury in a motor-car accident, without loss of consciousness. Thereafter she was largely confined to bed at home, but fell on several occasions when attempting to get up.

On May 15, 1965 she became suddenly unconscious and was admitted to hospital where she was found to be stuporose, cyanosed, and dyspnoeic. The pulse was of small volume and was not paradoxical; the blood pressure was $125 / 85 \mathrm{~mm}$. Hg. There was no peripheral oedema but non-pulsatile distension of jugular veins was noted. The apex beat was not palpable but the heart appeared to be enlarged on percussion and this was confirmed radiologically. A long high-pitched diastolic murmur was heard at the left sternal border. The appearances of the optic fundi were normal, the pupils were of equal size and moderately dilated, and there was a divergent strabismus. There was hyperreflexia of the left arm and leg but no other objective abnormality was found on neurological examination. The skull $x$-ray film was normal. The electrocardiogram showed right axis deviation and $T$ wave inversion in the praecordial leads. There was moderate proteinuria. Blood urea was $47 \mathrm{mg} . / 100 \mathrm{ml}$; blood sugar was $84 \mathrm{mg} .100 \mathrm{ml}$. The cerebrospinal fluid was normal in composition and was under normal pressure. Soon after admission the patient became more profoundly unconscious. Bilateral carotid angiography showed no displacement of the cerebral vessels. The blood pressure fell steadily despite supportive therapy and she died in deep coma some 16 hours after admission. Necropsy was performed 21 hours after death.

\section{Necropsy}

Heart (weight 500 g.). There was a little strawcoloured fluid in the pericardial sac and no evidence of pericarditis. A bulky tumour mass arose from the wall of the right ventricle and greatly diminished the right ventricular lumen. From the main tumour mass 


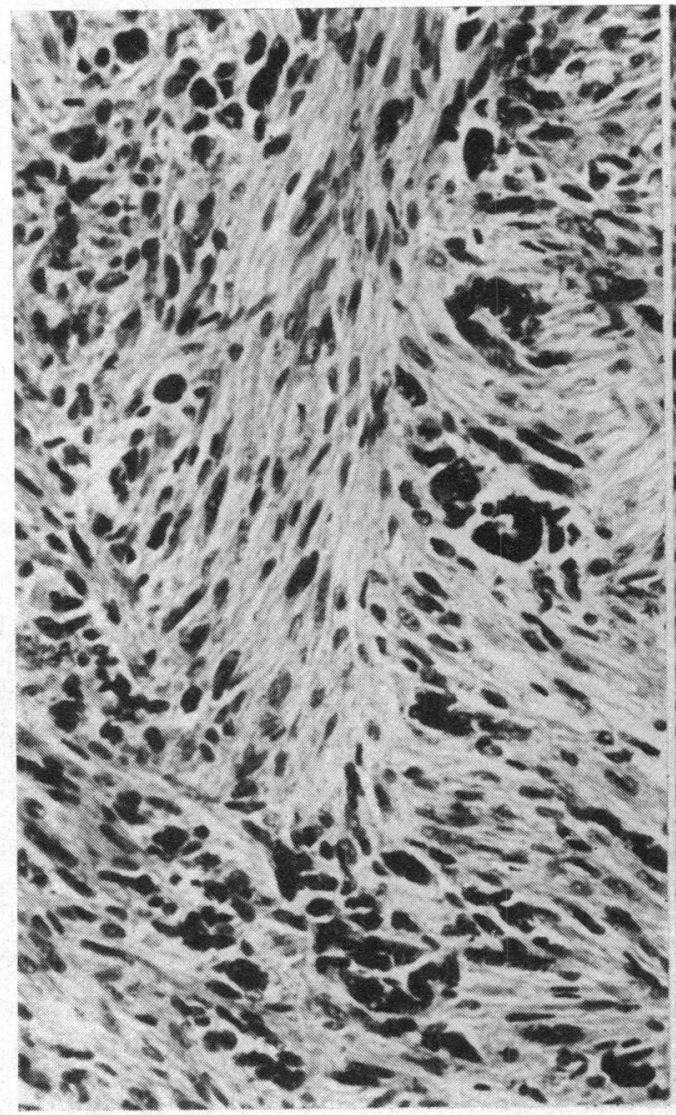

(a)

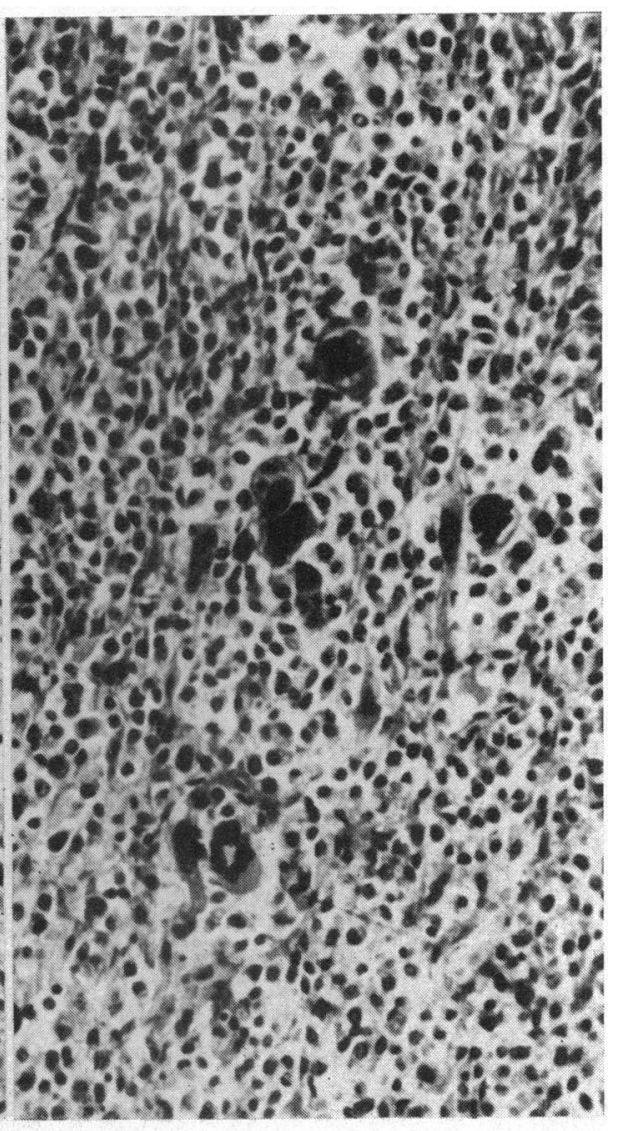

(b)

FIG. 1a. Uterine tumour. Note abnormally large cells with bizarre nuclei. $(H$. and $E$. $\times 210$.) FIG. 1b. Heart tumour. Small round and fusiform cells together with giant and multinucleate cells. (H. and E. $\times 210$.)

(approximately $9 \times 8 \times 6 \mathrm{~cm}$.) smooth glistening polypoid projections extended to involve both the tricuspid valve and the pulmonary conus, producing considerable obstruction to right ventricular outflow and impinging upon the pulmonary valve (Fig. 2). The tumour had directly invaded the interventricular septum to reach the left ventricle and penetrate the heart posteriorly. On section the tumour was of firm homogeneous consistency and slightly paler in colour than the normal myocardium.

Histology (Fig. 1b). The tumour was extremely cellular, mainly consisting of masses of small cells possessing a compact eosinophil cytoplasm and round, oval, or spindle-shaped nuclei. In areas there were numerous mitotic figures and many bizarre giant cells, some multinucleate. Elsewhere the architecture was more regular, and spindle-shaped cells, a few apparently containing myofibrils, were arranged in bundles. Special stains showed minimal collagen deposition; there was a very fine stroma, and a dense vasculature.
Cross-striation of muscle cells was not demonstrated. The tumour was regarded as a leiomyosarcoma.

Other Organs. There was congestion of the liver and spleen with moderate pulmonary oedema. The brain showed no evidence of cerebrovascular disease or other abnormality. Histological examination of pelvic vault tissue, bladder, left ovary, pelvic and abdominal lymph nodes, lungs, brain, bone-marrow, liver, and spleen revealed no evidence of tumour.

Review of Uterine Tumour. Review of sections confirmed the 1955 diagnosis of leiomyosarcoma. Furthermore, certain resemblances were noted between the heart and uterine lesions. The majority of the heart tumour cells were rather smaller and more compact than the cells of the uterine tumour, but the bizarre multinucleate and giant cell variants were similar, and staining characteristics identical. Thus, both heart and uterine neoplasms were leiomyosarcomas, and showed similarities which prompted consideration of a relationship between the two tumours. 
The heart tumour may have arisen as a primary neoplasm, or as a metastasis from the uterine tumour. Although an extremely rare finding at necropsy, leiomyosarcoma is among the more common primary malignant tumours of the heart (Prichard, 1951). If, however, this lesion is a primary tumour, the patient developed two unrelated malignant tumours of smooth muscle of closely similar appearance.

If the heart lesion is a secondary tumour, then a long period of time had elapsed since the primary leiomyosarcoma was removed and it seems surprising that metastasis should only have occurred to the heart. We have found only one previous report of metastasis of uterine leiomyosarcoma to the heart (Rosenblatt and Featherston, 1960), though these authors make reference to six other unreported cases. In their own case, hysterectomy also preceded the onset of cardiac symptoms, but the cardiac tumour was not the only salient finding at necropsy since tumour deposits were present in the left lung.
If the theories of Yater and of Windholz (quoted by Prichard, 1951) are correct, metastatic tumour emboli reach the right heart not by direct implantation from blood within the right ventricular lumen, but via the coronary arteries and veins. Thus, tumour emboli could only reach the myocardium of the right ventricle having passed through the lungs, and the complete absence of pulmonary tumour deposits in our case might be held to suggest that no such passage has taken place, and that the cardiac tumour is a primary lesion.

However, during the development of the extensive right ventricular lesion it seems almost certain that the lungs were subjected to tumour emboli originating from friable neoplastic processes in the right ventricle. No growth occurred, and it therefore seems likely that the lungs offered an unfavourable soil for the cells of this particular tumour; tiny tumour emboli might have traversed the lung without successful implantation, to settle ultimately in the myocardium, and absence of 
pulmonary deposits does not exclude the possibility that the heart tumour is secondary to the uterine lesion.

The similarities between the histological appearances of heart and uterine leiomyosarcomata have already been mentioned. However, neither these nor other considerations are sufficient to justify the firm conclusion that the heart tumour was a secondary deposit. Indeed, a number of senior pathologists have been asked to review the slides and to consider the case, and opinions are equally divided as to whether the cardiac tumour is primary or secondary.

Cerebral features were prominent in this patient's terminal illness; it was thought that they might be due to a subdural haematoma caused by the recent head injury, and the possibility of a late cerebral metastasis from the uterine tumour was also considered. It is clear that insufficient importance was attached to the raised venous pressure. The symptoms of weakness and tiredness and latterly the neurological abnormalities with stupor and coma were probably caused by the very low fixed cardiac output due to serious interference with right ventricular filling by the bulky tumour mass. Such a presentation, with clinical features resembling those of constrictive pericarditis, is not uncommon in intracardiac neoplasms (Emanuel and Lloyd, 1962). At necropsy, processes of tumour were observed to impinge upon the pulmonary valve, clearly impairing its competence, and this had given rise to the diastolic murmur noted clinically.

In addition to intracardiac obstruction and interference with valve function, cardiac tumours may lead to disturbances of conduction or rhythm, or may give rise to signs of pericarditis (Gassman, Meadows, and Baker, 1955). The present case demonstrates that severe restriction of cardiac output may result in presentation with predominantly neurological features.

\section{Summary}

A patient died as a result of massive involvement of the right ventricle by a leiomyosarcoma. A similar tumour, arising in the uterus, had been removed almost 10 years before, and the possible relationship between the two tumours is discussed. Clinically the patient presented with predominantly neurological features and the final diagnosis was made at necropsy.

We are grateful to Dr T. N. Morgan and Professor A. R. Currie for their help in the preparation of this paper. We are also grateful to Professor A. C. Lendrum and Professor R. A. Willis for their opinion of the pathology.

\section{References}

Emanuel, R. W., and Lloyd, W. E. (1962). Right atrial myxoma mistaken for constrictive pericarditis. Brit. Heart F., 24, 796.

Gassman, H. S., Meadows, R., Jr., and Baker, L. A. (1955). Metastatic tumors of the heart. Amer. F. Med., 19, 357.

Prichard, R. W. (1951). Tumors of the heart. Arch. Path., 51, 98.

Rosenblatt, W. H., and Featherston, W. P. (1960). Metastatic leiomyosarcoma of the heart. Amer. F. Cardiol., 6, 672. 